\title{
Dark Matter Phenomenology from Upcoming Neutrino Telescopes
}

\section{Andrew Cheek, ${ }^{a, *}$ S. Basegmez du Pree, ${ }^{b}$ C. Arina, ${ }^{a}$ A. Dekker, ${ }^{c}$ M. Chianese ${ }^{d, e}$ and S. Ando ${ }^{c, f}$}

${ }^{a}$ Centre for Cosmology, Particle Physics and Phenomenology (CP3), Université catholique de Louvain, Chemin du Cyclotron 2, B-1348 Louvain-la-Neuve, Belgium

${ }^{b}$ Nikhef, National Institute for Subatomic Physics, Science Park 1051098 XG, Amsterdam, The Netherlands

${ }^{c}$ GRAPPA Institute, University of Amsterdam, 1098 XH Amsterdam, The Netherlands

${ }^{d}$ Dipartimento di Fisica "Ettore Pancini", Università degli studi di Napoli "Federico II", Complesso Univ. Monte S. Angelo, I-80126 Napoli, Italy

${ }^{e}$ INFN - Sezione di Napoli, Complesso Univ. Monte S. Angelo, I-80126 Napoli, Italy

${ }^{f}$ Kavli Institute for the Physics and Mathematics of the Universe (Kavli IPMU, WPI), University of Tokyo, Kashiwa, Chiba 277-8583, Japan

E-mail: andrew. cheek@uclouvain. be

Experimental developments in neutrino telescopes are drastically improving their ability to constrain the annihilation cross-section of dark matter. Focusing on particle models for dark matter, we assess how these future limits will complement the existing landscape of dark matter searches. We bring together results from gamma-ray telescopes, measurements of the cosmic microwave background and direct dark matter detection. Our projections are based on the Angular Power Spectrum method, which is a powerful tool for reducing astrophysical uncertainties. We find that neutrino telescopes will be able to competitively probe significant portions of parameter space and will provide critical complementary information to the search for dark matter. Furthermore, we identify models that can potentially be explored where the relic abundance is achieved through thermal freeze-out. This proceeding is based on Ref [1].

$37^{\text {th }}$ International Cosmic Ray Conference (ICRC 2021)

July 12 th - 23rd, 2021

Online - Berlin, Germany

\footnotetext{
*Presenter
} 


\section{Introduction}

The properties and origin of dark matter is one of the most pursued problems in astroparticle physics today. With tremendous advances in experimental sensitivities of $\gamma$-ray telescopes, direct detection experiments and collider searches, many first order particle physics explanations for dark matter have been abandoned. Despite this, the next generation of neutrino telescopes will probe a significant region of uncharted parameter space for dark matter annihilation and decay into neutrinos [2]. In Ref. [1] we explored the dark matter models that these new experiments would competitively probe.

The neutrino experiments in this next generation are; IceCube Upgrade [3], IceCube-Gen 2 [4], Baikal-GVD [5], P-ONE [6] and KM3NeT [7]. The choice made is Ref. [1] was to focus on the potential of KM3NeT, which comprises of a cubic kilometer of optical arrays, already in partial operation in the Mediterranean Sea. We made this choice because KM3NeT will have a superior angular resolution as well as having a good field of view to the galactic centre, but the results shown here can be readily applied to similar detectors.

The projections for KM3NeT that we calculated are not necessarily the most optimistic as such limits are often analysis dependent. This is because we opted to employ an angular power spectrum (APS) analysis [8,9], which is remarkably robust against changes in the dark matter density profile. This of course comes at a cost, and we anticipate with a different analysis, projections assuming a profile which is denser in the centre of the Galaxy, could be more sensitive. The important point is however, that there is a large degree of uncertainty associated with the dark matter profile, specifically in the central regions of the Galaxy. It is important to develop methods, such as the APS, which help minimize the effects of such uncertainties.

Equipped with our projects we then interpret them in terms of simple particle physics models. In this proceedings, we briefly discuss how simple models will inevitably provoke a rich phenomenology, making it difficult to evade existing bounds and producing sizable signals in neutrino telescopes. However, there is an interesting case for a secluded $U(1)_{L_{\mu}-L_{\tau}}$ mediated dark matter model. For this simple and well motivated model, neutrino telescopes will provide a unique probe in interesting regions of parameter space.

\section{Angular Power Spectrum}

Here we briefly summarize the method we use to obtain our projections for 10 years of data taking in the KM3NeT-ARCA site [7]. We employ an angular power spectrum (APS) analysis described in Refs. [8,9] where the anisotropy of the expected dark matter signal coming from the Galactic center is exploited in order to distinguish against the approximately isotropic astrophysical and atmospheric backgrounds. Our analysis extends to a lower energy region from that in ref. [9], which limited its study to $m_{\mathrm{DM}} \geq 200 \mathrm{TeV}$, here our projections start at $m_{\mathrm{DM}}=200 \mathrm{GeV}$. In this energy region, the background neutrino fluxes that dominate are from atmospheric neutrinos.

Once we generate a database of neutrino skymaps with dark matter signals and expected background, we can expand said skymaps in spherical harmonics. By taking the average expansion coefficients for a each moment, $C_{\ell}$, for each skymap, we can calculate a $\chi_{\mathrm{md}}^{2}$ for the background only hypothesis and use that to determine the $p$-value for a signal skymap. Then, for a given dark 

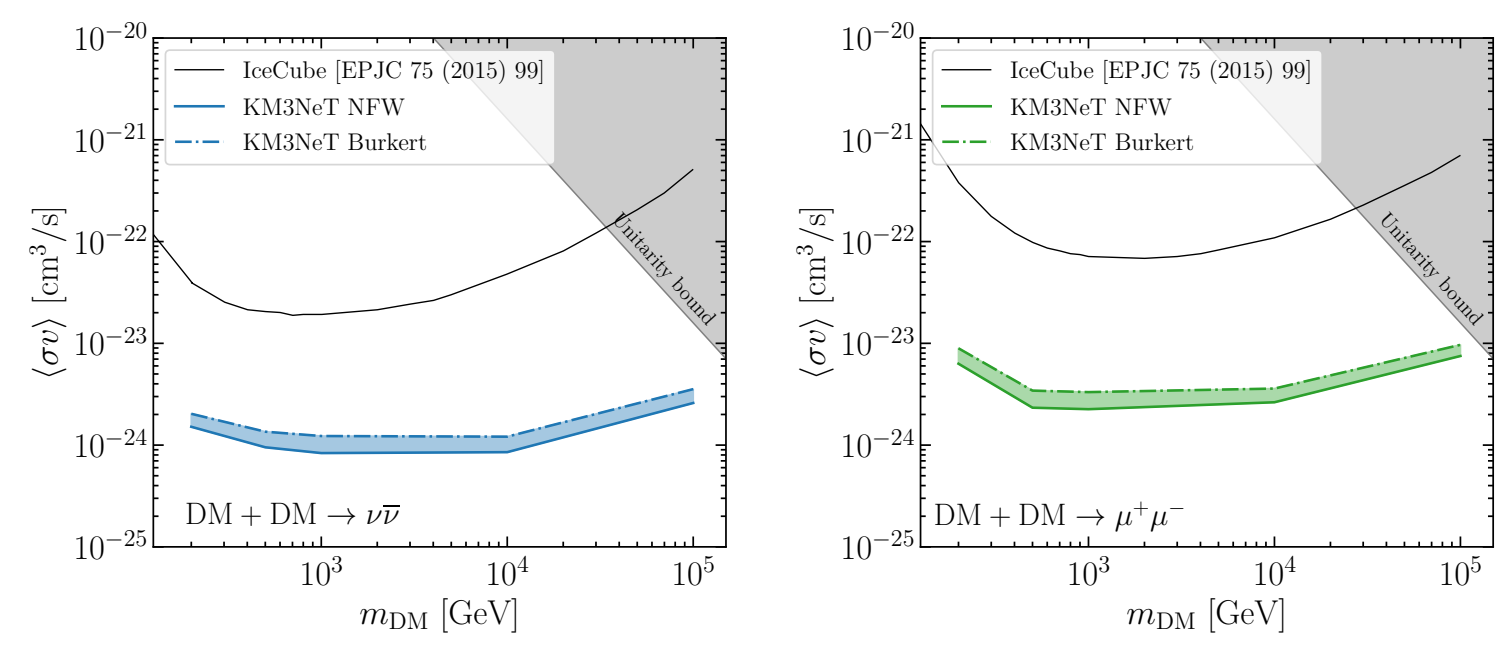

Figure 1: Left: Present and future limits on the dark matter annihilation cross-section placed by APS analyses of neutrino telescope data. The bands represent the uncertainty related to different galactic dark matter profile: the lower (upper) edges refer to NFW (Burkert) density profile. The colored shaded region is the median sensitivity at $90 \% \mathrm{CL}$ after 10-year exposure of KM3NeT obtained through the angular power spectrum (APS) method in case of NFW (solid lines) and Burkert (dot-dashed lines) profiles. The black band corresponds to a 1-year IceCube with a similar multipole study [8]. The grey region in the top-right corner is excluded by unitarity (see text). Right: Same as left for dark matter annihilation into muons.

matter mass $m_{\mathrm{DM}}$, we calculate the annihilation cross-sections $\langle\sigma v\rangle$ that will be excluded to $90 \%$ confidence level (CL).

In figure 1 we present our projected limits for annihilation purely into neutrinos (left) and muons (right). We show the projections for both the Navarro-Frenk-White (solid line) and Burkert (dot-dashed line) distributions. We do this to exhibit how robust the APS analysis is with respect to changed in the halo profile. We also show the results from a previous APS study using existing IceCube data from only 1 year of data taking [8].

\section{Dark matter models and existing constraints}

Now that we have our model independent projections, we can assess what the implications are for specific particle models for dark matter. We specifically are interested in simple models that only extend the Standard Model (SM) by two particles, a mediator and and dark matter candidate, see ref. [10] for a review of models. Attempts have been made to connect the dark matter to neutrinos via a sterile neutrino portal [11], we do not consider this because constraints on active-sterile neutrino mixing mean that perturbative couplings are unreachable for $m_{\mathrm{DM}} \gtrsim 100 \mathrm{GeV}$.

Naturally, since we are interested in neutrino telescopes, we focus on models than only couple to leptons (leptophilic). Due to the group structure of the SM, annihilation of dark matter into neutrinos is generically accompanied by annihilation into charged leptons. Therefore, $\gamma$-ray constraints are important to consider. We take the limits for a continuum gamma-ray spectrum provided by the Fermi-LAT bounds from dwarf spheroidal galaxies (dSphs) [12]. We calculate the limits at 90\% CL using the likelihood method implemented within MADDM [13]. 
Furthermore, leptophilic models are also constrained by direct dark matter detection experiments through loop processes. This leads to quite sizable constraints coming from existing experiments. The strongest of which, XENON1T [14], is what we compare to.

In ref. [1] we consider simplified models for a Dirac dark matter $(\chi)$ mediated by a charged scalar $(\varphi)$ or a vector boson $\left(Z^{\prime}\right)$. For the scalar mediator, the branching ratio to neutrinos and charged leptons is $50 \%$ respectively. This should be enough for our projections to be much more sensitive to the model than the $\gamma$-ray bounds, however, since $\langle\sigma v\rangle \propto \frac{m_{\chi}^{2}}{\left(m_{\chi}^{2}+m_{\varphi}^{2}\right)^{2}}$ and $m_{\varphi}>m_{\chi}$ for DM stability, the cross-section at high masses is limited by perturbativity of couplings. The situation can be improved somewhat if $m_{\chi} \sim m_{\varphi}$, however, even in the limiting case where couplings are a the limiting value of $4 \pi$, direct detection constraints are the strongest for the vast majority of parameter space.

For the vector mediator, a resonant enhancement takes place for the cross-section when $m_{Z^{\prime}} \sim$ $2 m_{\chi}$. This means that our KM3NeT projections will be able to probe significant new regions of parameter space. Additionally, now it is possible that $m_{Z^{\prime}}<m_{\chi} . U(1)$ extensions of the SM are not unlimited by theoretical considerations. For example, axial vector couplings lead to unitarity violation. This is related to the fact that this model would not be anomaly free.

One of the simplest anomaly-free extensions to the SM is the $U(1)_{L_{\alpha}-L_{\beta}}$ where $\alpha$ and $\beta$ denote generation. Of which $U(1)_{L_{\mu}-L_{\tau}}$ is currently less constrained experimentally than models containing interactions with electrons. We can add a vector-like fermion $\chi$, as our dark matter candidate without introducing any anomalies. In figure 2 we show how our projections stack up against existing bounds for both $m_{Z^{\prime}} \sim m_{\chi}$ (left) and $m_{Z^{\prime}} \ll m_{\chi}$ (right).

On the left panel of figure 2 we see how both the Fermi-LAT (grey) and KM3NeT (green) constraints are strongest on the resonance. Interactions between $Z^{\prime}$ and nuclei come from the loop induced kinetic mixing and can provoke a signal in XENON1T (light blue). In pink we also display the preferred region where this new boson can accommodate flavor anomalies observed at LHCb [19]. However, in order for this model to account for anomalies in $b \rightarrow s \mu^{+} \mu^{-}$decays, it is necessary to assume that there is additional new physics.

In the case where $m_{Z^{\prime}} \ll m_{\chi}$, collider, precision and direct detection experiments are highly constraining, hence we consider $g_{\mathrm{SM}} \ll g_{\mathrm{DM}}$. Both $m_{Z^{\prime}}$ and $g_{\mathrm{SM}}$ are constrained from below however. The effective relativistic degree of freedom before recombination, $\Delta N_{\text {eff }}$, sets a bound on the $Z^{\prime}$ mass to be larger than $4 \mathrm{MeV}$ [18]. While $g_{S M}$ has to be large enough such that Big Bang Nucleosynthesis $(\mathrm{BBN})$ is not disrupted. sets a lower bound on the coupling strength not to spoil its accurate predictions in terms of element abundances with the injection of energy. Indeed the $\chi \chi \rightarrow Z^{\prime} Z^{\prime}$ process is independent to $g_{\mu-\tau}$, however one cannot choose an arbitrarily small value as a sufficiently long lived $Z^{\prime}$ will disrupt BBN. A conservative bound would be taking the lifetime sufficiently shorter than $\sim 1 \mathrm{~s}$. For the masses shown on the right of figure $2, g_{\mathrm{SM}}$ can always be chosen such that the future LZ direct detection experiment will not be sensitive to it and BBN will not be disrupted.

With such a scale difference between $m_{\chi}$ and $m_{Z^{\prime}}$ the dominant annihilation is now $\chi \bar{\chi} \rightarrow Z^{\prime} Z^{\prime}$. Furthermore $m_{Z^{\prime}}$ can act as a long range force such that Sommerfeld effects enhance $\langle\sigma v\rangle$. This effect is more pronounced in environments where dark matter is slower. Therefore, constraints from Planck [17] are more stringent than Fermi-LAT. We see on the right of figure 2 that the 



Figure 2: Left: The projected sensitivity of KM3NeT (green) together with current bounds from Fermi-LAT dSphs obtained with MADDM (grey) [12] and XENON1T (light blue) [14] obtained with RAPIDD [15], as labelled, in the $\left\{m_{\chi}, m_{Z^{\prime}}\right\}$-plane for the anomaly-free $U(1)_{L_{\mu}-L_{\tau}}$ model. The black dotted lines are where $\langle\sigma v\rangle=2 \times 10^{-26} \mathrm{~cm}^{3} / \mathrm{s}$, i.e. the value for simple freeze-out. All exclusion limits and projection are provided at $90 \% \mathrm{CL}$. The pink shaded region is the favored region [16] for explaining the flavour anomalies found at LHCb. Right: The projected sensitivity of KM3NeT (green) together with current upper bound from Planck (blue) [17], as labelled, in the $\left\{m_{\chi}, m_{Z^{\prime}}\right\}$-plane for fixed couplings of $g_{\mu-\tau}<<g_{\chi}=1$. The yellow region denotes the model parameter space favoured to alleviate the Hubble tension [18]. The dotted black line represents the relic density line.

Planck constraints fall off dramatically for $m_{Z^{\prime}}<2 m_{\mu}$, this is because the decays of $Z^{\prime}$ into muons in no longer kinematically allowed, leading to a branching ratio to neutrinos $\approx 100 \%{ }^{1}$. We see in this region that neutrino telescopes would be uniquely placed to probe such a scenario. Interestingly, thanks to the Sommerfeld effect, $\langle\sigma v\rangle$ will be enhanced in the galactic center such that our projections for KM3NeT will be able to probe the thermal relic line inferred from thermal freeze-out. Furthermore, in this region, the yellow band in figure 2 signifies the values where $m_{Z^{\prime}}$ could contribute enough to $\Delta N_{\text {eff }}$ which is a possible resolution to the Hubble tension.

\section{Conclusion}

In this proceedings we have reviewed the work of ref. [1], which explores possible dark matter models that upcoming neutrino telescopes will probe. We have done this in a global way, considering also other searches which provide complimentary constraints. By doing so we identified, well motivated and simple dark matter models that will be uniquely probed by neutrino telescopes.

\footnotetext{
${ }^{1}$ The branching ratio to electrons through kinetic mixing is non-zero but around $0.001 \%$.
} 


\section{References}

[1] S. Basegmez Du Pree, C. Arina, A. Cheek, A. Dekker, M. Chianese and S. Ando, Robust Limits from Upcoming Neutrino Telescopes and Implications on Minimal Dark Matter Models, JCAP 05 (2021) 054 [2103.01237].

[2] C.A. Argüelles, A. Diaz, A. Kheirandish, A. Olivares-Del-Campo, I. Safa and A.C. Vincent, Dark Matter Annihilation to Neutrinos, 1912.09486.

[3] ICECube collaboration, Dark matter searches with the IceCube Upgrade, PoS ICRC2019 (2020) 506 [1908.08236].

[4] ICECUBE collaboration, Neutrino astronomy with the next generation IceCube Neutrino Observatory, 1911.02561.

[5] A.D. Avrorin et al., Sensitivity of the Baikal-GVD neutrino telescope to neutrino emission toward the center of the galactic dark matter halo, JETP Lett. 101 (2015) 289 [1412 . 3672].

[6] P-ONE collaboration, The Pacific Ocean Neutrino Experiment, Nature Astron. 4 (2020) 913 [2005.09493].

[7] S. Adrián-Martínez, M. Ageron, F. Aharonian, S. Aiello, A. Albert, F. Ameli et al., Letter of intent for KM3net 2.0, Journal of Physics G: Nuclear and Particle Physics 43 (2016) 084001.

[8] ICECuBE collaboration, Multipole analysis of IceCube data to search for dark matter accumulated in the Galactic halo, Eur. Phys. J. C 75 (2015) 20 [1406.6868].

[9] A. Dekker, M. Chianese and S. Ando, Probing dark matter signals in neutrino telescopes through angular power spectrum, JCAP 09 (2020) 007 [1910 . 12917].

[10] C. El Aisati, C. Garcia-Cely, T. Hambye and L. Vanderheyden, Prospects for discovering a neutrino line induced by dark matter annihilation, JCAP 10 (2017) 021 [1706.06600].

[11] M. Blennow, E. Fernandez-Martinez, A. Olivares-Del Campo, S. Pascoli, S. Rosauro-Alcaraz and A. Titov, Neutrino Portals to Dark Matter, Eur. Phys. J. C 79 (2019) 555 [1903. 00006].

[12] DES, Fermi-LAT collaboration, Searching for Dark Matter Annihilation in Recently Discovered Milky Way Satellites with Fermi-LAT, Astrophys. J. 834 (2017) 110 [1611.03184].

[13] F. Ambrogi et al., MadDM v.3.0: a Comprehensive Tool for Dark Matter Studies, Phys. Dark Univ. 24 (2019) 100249 [1804.00044].

[14] XENON collaboration, Dark Matter Search Results from a One Ton-Year Exposure of XENONIT, Phys. Rev. Lett. 121 (2018) 111302 [1805.12562].

[15] D. Cerdeno, A. Cheek, E. Reid and H. Schulz, Surrogate Models for Direct Dark Matter Detection, JCAP 08 (2018) 011 [1802.03174]. 
[16] W. Altmannshofer, S. Gori, S. Profumo and F.S. Queiroz, Explaining dark matter and B decay anomalies with an $L_{\mu}-L_{\tau}$ model, JHEP 12 (2016) 106 [1609. 04026].

[17] T.R. Slatyer, Indirect dark matter signatures in the cosmic dark ages. I. Generalizing the bound on s-wave dark matter annihilation from Planck results, Phys. Rev. D 93 (2016) 023527 [1506.03811].

[18] M. Escudero, D. Hooper, G. Krnjaic and M. Pierre, Cosmology with A Very Light $L_{\mu}-L_{\tau}$ Gauge Boson, JHEP 03 (2019) 071 [1901. 02010].

[19] LНСв collaboration, Search for lepton-universality violation in $B^{+} \rightarrow K^{+} \ell^{+} \ell^{-}$decays, Phys. Rev. Lett. 122 (2019) 191801 [1903.09252]. 